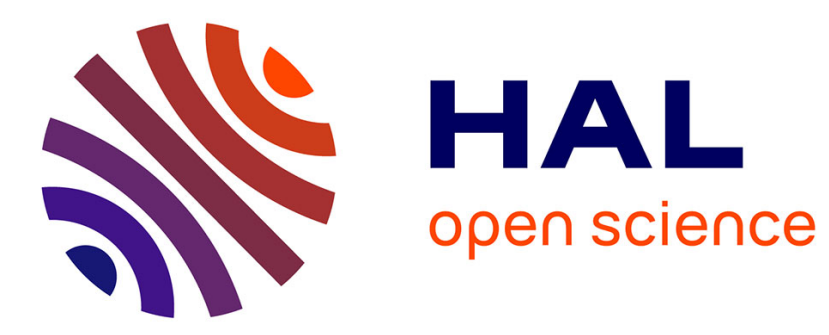

\title{
Quantifying uncertainties on the solution model of seismic tomography
}

Carole Duffet, Delphine Sinoquet

\section{To cite this version:}

Carole Duffet, Delphine Sinoquet. Quantifying uncertainties on the solution model of seismic tomography. Inverse Problems, 2006, 22 (2), pp.525-538. 10.1088/0266-5611/22/2/009 . hal-02284112

\section{HAL Id: hal-02284112 \\ https://hal-ifp.archives-ouvertes.fr/hal-02284112}

Submitted on 11 Sep 2019

HAL is a multi-disciplinary open access archive for the deposit and dissemination of scientific research documents, whether they are published or not. The documents may come from teaching and research institutions in France or abroad, or from public or private research centers.
L'archive ouverte pluridisciplinaire HAL, est destinée au dépôt et à la diffusion de documents scientifiques de niveau recherche, publiés ou non, émanant des établissements d'enseignement et de recherche français ou étrangers, des laboratoires publics ou privés. 


\title{
Quantifying uncertainties on the solution model of seismic tomography
}

\author{
Carole Duffet $^{1,2}$ and Delphine Sinoquet ${ }^{2}$ \\ ${ }^{1}$ Laboratoire ACSIOM, Département de Mathématiques, CC051, Université de Montpellier II, \\ Place Eugène Bataillon, 34095 Montpellier II Cedex, France \\ 2 Technology, Computer Science, and Applied Mathematics Division, Institut Français du \\ Pétrole, 1 \& 4 avenue de Bois, Préau, 92852 Rueil-Malmaison, France \\ E-mail: duffet@math.univ-montp2.fr
}

Received 3 October 2005, in final form 23 February 2006

Published 15 March 2006

Online at stacks.iop.org/IP/22/525

\begin{abstract}
Reflection tomography allows the determination of a propagation velocity model that fits the traveltime data associated with reflections of seismic waves in the subsurface. A least-squares formulation is used to compare the observed traveltimes and the traveltimes computed by the forward operator based on a ray tracing. The solution of this inverse problem is only one among many possible models. A linearized a posteriori analysis is then crucial to quantify the range of admissible models we can obtain from these data and the a priori information. The contribution of this paper is to propose a formalism which allows us to compute uncertainties on relevant geological quantities for a reduced computational time. Nevertheless, this approach is only valid in the vicinity of the solution model (linearized framework), complex cases may thus require a nonlinear approach. Application on a $2 \mathrm{D}$ real data set illustrates the linearized approach to quantify uncertainties on the solution of seismic tomography. Finally, the limitations of this approach are discussed.
\end{abstract}

(Some figures in this article are in colour only in the electronic version)

\section{Introduction}

Geophysical methods for imaging complex geological subsurfaces in petroleum exploration requires the determination of an accurate propagation velocity model. Seismic reflection tomography (see [2]) turns out to be an efficient method for that: this method allows us to determine a seismic velocity distribution from traveltime data associated with the seismic waves reflecting on geological surfaces. This inverse problem is formulated as a leastsquares minimization problem which consists of the minimization of the mismatch between the observed traveltimes and the traveltimes computed by the forward problem (solved by 
a ray tracing method). The first section of this paper presents an overview of the reflection tomography method we have developed (see [14-16, 20] for more details).

In principle, the estimation of the uncertainties begins with an analysis of the errors on the solution model by applying Monte Carlo techniques among others (for examples see [18, 17]). These methods have a prohibitively expensive computational cost. Our goal here is to develop methods for the a posteriori analysis of the solution which will be easier to apply and nevertheless provide useful results. Classically, the analysis of the solution consists only of checking the misfits between observed traveltimes and calculated traveltimes by computing characteristic values of the misfit distribution and by studying the spatial distribution of the misfits. This analysis is not sufficient: even if these quality control criteria are matched, the determined model is only one of many possible models that match the data. An uncertainty analysis should be performed to quantify the range of admissible models we can obtain from these data and the a priori information (see [10]).

The different methods we are interested in here allow us to access the uncertainties on the solution model thanks to the analysis of the a posteriori covariance matrix obtained in the linearized framework (see $[11,21]$ among others). The computation of this matrix is generally expensive for 3D problems; among others, [1] proposed a method to compute approximations of the covariance and resolution matrices from the different iterates when the Gauss-Newton linear system is solved by an iterative method such as the gradient conjugate algorithm. But the physical interpretation of the terms of this huge matrix may be cumbersome when we want to go further than the classical but restrictive analysis of the diagonal terms. In this paper, we propose another approach which simulates admissible models from an a posteriori probability density function that directly provides physical models. This method requires a Cholesky decomposition of the Gauss-Newton matrix which may be unfeasible for 3D realistic models. To overcome this difficulty, we propose a formalism based on linear combinations of model parameters (macro-parameters) that allows us to compute uncertainties on relevant geological quantities (the average thickness of a layer for example) for a reduced computational time (the a posteriori covariance matrix is reduced to the macro-parameter space). The application of these methods on two real data sets shows their effectiveness and their limitations are discussed, particularly the limitations due to the linearization: a nonlinear approach is proposed in order to delimit the space of admissible solutions.

In this paper, after the description of the chosen formulation of reflection tomography in section 2, we present the two proposed methods in section 4 to perform a linearized uncertainty analysis on the solution model of a $2 \mathrm{D}$ real data set described in section 3 . In section 5 , we explain the motivations to carry out a nonlinear approach on this $2 \mathrm{D}$ complex case and present some results. Finally, section 6 is devoted to concluding the paper.

\section{The reflection tomography problem}

Let us first present the reflection tomography method which was developed in the KIM consortium [16]. The chosen model representation is a blocky velocity model where the velocity distribution is described by slowly varying layer velocities (also called velocity blocks) delimited by interfaces (see figure 1 for an example). The model is thus composed of two kinds of parameters: parameters describing the velocity variations within the layers and parameters describing the geometry of the interfaces delimiting the velocity blocks ${ }^{3}$. Moreover, anisotropy of the velocity may be modelled by two parameters $\eta$ and $\delta$ (see [20]

3 In our approach (see Jurado et al [14]), the subsurface model $m$ is composed of 2D or 3D B-spline functions describing velocity variations in a layer $(v(x, y)+k . z$ or $v(x, y, z))$ and $2 \mathrm{D} \mathrm{B}$-spline functions describing the interfaces $(Z(x, y), Y(z, x)$ or $X(y, z))$ (see [6, 13]). 


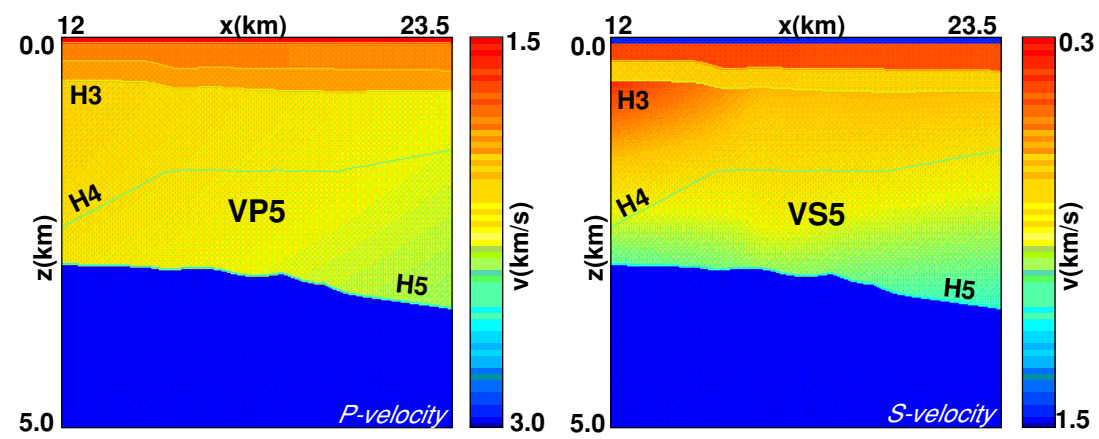

Figure 1. Solution velocity model obtained by tomography. Left: P velocity. Right: S velocity. The RMS value of the traveltime misfits is $6.2 \mathrm{~ms}$. The anisotropy parameters values are $\eta=6.29 \%$ and $\delta=-4.43 \%$.

for more details). This blocky representation allows the representation of complex models (e.g., presenting rapid lateral velocity variations and complex geological structures) with a reasonable number of unknowns. Strong a priori information is introduced, the number of layers being fixed: this reduces the underdetermination of the inverse problem.

The forward problem of reflection tomography consists of computing traveltimes of reflected seismic waves, given a model, an acquisition survey (locations of the sources and the receivers) and signatures (generally reflectors where the waves reflect). It is solved by a ray tracing method which is a high frequency approximation of the wave equation $($ see $[5,15]$ among others). We denote by $T(m)$ the forward modelling operator, that gives, for a specified reflector, the traveltimes associated with all source-receiver pairs.

Reflection traveltime tomography is the corresponding inverse problem: its purpose is to adjust $m$ such that $T(m)$ best matches a vector of traveltimes $T^{\text {obs }}$ picked on seismic data. A natural formulation of this problem is the least-squares formulation

$$
\left\|T(m)-T^{\mathrm{obs}}\right\|_{C_{D}^{-1}}^{2}+\left\|m-m^{\text {prior }}\right\|_{C_{M}^{-1}}^{2},
$$

where

- $\|-\|_{A}$ denotes the norm defined by $A$ matrix: $\|x\|_{A}=x^{T} A x$.

- $C_{D}$ is the a priori covariance operator in the data space that allows us to describe errors on the data,

- $m^{\text {prior }}$ is an a priori model (coming, for instance, from geological knowledge and/or additional information resulting from well measurements),

- $C_{M}$ is the a priori covariance matrix in the model space that describes errors on the a priori model.

In practice, building an a priori model is difficult and the a priori term, which is necessary to well pose the inverse problem, is often replaced by regularization terms made up of second derivatives of the model (see for instance [9]) which leads to $C_{M}^{-1}=\mathcal{Q}_{\text {reg }}$ and $m^{\text {prior }}=0$

$$
\left\|T(m)-T^{\mathrm{obs}}\right\|_{C_{D}^{-1}}^{2}+\epsilon^{2}\left\|D^{2} m\right\|_{\mathrm{Id}}^{2}
$$

where a continuation technique (see [4]) is applied for tuning the regularization weight $\epsilon$ : the weight is decreased until the model matches the data with the expected accuracy. The obtained model is then the smoothest model which fits the data. This methodology allows us to stabilize the inversion. 
This large size (up to $\sim 100000-500000$ traveltime data and $\sim 5000-10000$ unknowns) nonlinear least-squares problem (the objective function is not quadratic since the forward modelling operator $T(m)$ is nonlinear) is solved classically by a Gauss-Newton method based on successive linearizations of the forward operator which needs the computation of the Jacobian matrix of $T\left(J(m)=\frac{\partial T}{\partial m}(m)\right) .{ }^{4}$ The resulting approximation of the Hessian matrix of the cost function is noted $H(m)=J(m)^{t} C_{D}^{-1} J(m)$.

Then, the quadratic approximation of the objective function (2)

$$
\tilde{C}_{n}(\delta m)=\left\|J_{n} \delta m-\delta T_{n}^{\mathrm{obs}}\right\|_{C_{d}^{-1}}^{2}+\epsilon^{2}\left\|D^{2}\left(m_{n}+\delta m\right)\right\|^{2}
$$

is minimized at each Gauss-Newton iteration $n$ by a preconditioned conjugate gradient $\left(m_{n}\right.$ is the current model, $\delta m$ is the unknown model perturbation, $J_{n}=\frac{\partial T}{\partial m}\left(m_{n}\right)$ is the Jacobian matrix evaluated at $m_{n}$ and $\delta T_{n}^{\mathrm{obs}}=T^{\mathrm{obs}}-T^{\mathrm{cal}}\left(m_{n}\right)$ ) (see [7]).

\section{Presentation of the application}

Let us consider a 2D real data set already studied by [3] with PP and $\mathrm{PS}^{5}$ data to illustrate all the following. 45338 traveltime data were interpreted and an uncertainty of $5 \mathrm{~ms}$ (respectively $8 \mathrm{~ms}$ ) is associated with PP data (respectively PS data). A layer-stripping approach (separate inversion of each velocity layer from the shallowest layer to the deepest one) provided the velocity model of figure 1 .

The model is described by four interfaces, corresponding to the interpreted events ( $H 1, H 3, H 4$ and $H 5)$ which define three layers with only lateral velocity variations for the first two uppermost layers ( $v(x)$, there is no vertical variations) and the deepest layer stretching from $H 3$ to $H 5$ with a 2D velocity $\operatorname{VP5}(x, z)$ and $V S 5(x, z)$ (figure 1). VP5 and VS5 define respectively the vertical velocity propagation of the $\mathrm{P}$-waves and the velocity propagation of the S-waves. This model is composed of 4588 parameters, 592 for the interfaces and 3936 for the velocities. In the deepest layer, we assume a vertical transverse isotropic velocity field characterized by VP5 and VS5 and the two parameters $\eta$ and $\delta$ measuring respectively the measure of the velocity an-ellipticity and the near vertical velocity propagation of the P-waves ${ }^{6}$. The values of $\eta$ and $\delta$ in the model correspond to a strongly anisotropic medium. For the first two layers, the inversion results are very satisfactory, with a traveltime misfit RMS (root mean square) of $3.5 \mathrm{~ms}$ for the PP-data and of $7 \mathrm{~ms}$ for the PS-data. For the last layer, the traveltime misfit RMS is of $3.7 \mathrm{~ms}$ and of $5.6 \mathrm{~ms}$ for, respectively, $H 4$ and $H 5$ PP-data and of $8.5 \mathrm{~ms}$ for $H 5$ PS-data, results which are consistent with the data uncertainties (figure 2).

As already shown by [20], it turns out that the anisotropy parameter $\delta$ is strongly undetermined from seismic data. The value of $\delta$ parameter was obtained in [3] by a trial and error approach in order to match approximately the depth of $H 5$ horizon given at well location.

4 The computation of the derivatives of $T$ with respect to the model parameters is cheap, thanks to the Fermat principle (see [2]). Thus, the derivatives of traveltimes with respect to velocity parameters are calculated by integrating a perturbation of the velocity along the rays calculated in the background model. The derivatives with respect to interface parameters are the integration of the background velocity along the shortened (negative contribution) or extended (positive contribution) part of the ray resulting from the interface displacement (we consider only the vertical displacement of impact point). See [16] for details.

5 PS-wave results from the conversion at the reflector of a down-going P-wave (compressional wave) into an up-going $\mathrm{S}$-wave (shear wave). In opposition to the PS-wave, the pure P mode is often called the PP-wave.

6 The expressions of $\eta$ and $\delta$ are $\delta=\frac{\left(C_{13}+C_{44}\right)^{2}-\left(C_{33}-C_{44}\right)^{2}}{2 C_{33}\left(C_{33}-C_{44}\right)}$ and $\eta=\frac{\epsilon-\delta}{1+2 \delta}$ where $\epsilon=\frac{C_{11}-C_{33}}{2 C_{33}}, C_{i j}$ being the elements of the elastic stiffness tensor $C$, which defines the linear relationships between the stress tensor and the strain tensor (following [22] and [20]). 


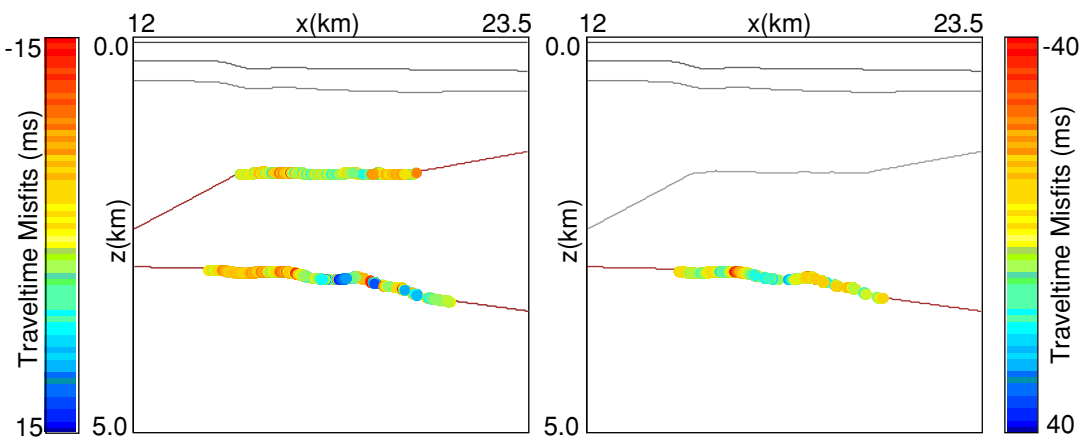

Figure 2. Interfaces of the solution velocity model obtained by tomography and their associated traveltime misfits: impact points of rays reflected on $H 4$ and $H 5$ (Left: PP rays. Right: PS rays), scales represent traveltime misfits. Note that the illuminated zone extends from $14 \mathrm{~km}$ to $20.5 \mathrm{~km}$.

We propose to carry on an a posteriori uncertainty analysis in order to quantify the uncertainties on this solution model: we focus on the anisotropic layer delimited by $H 3$ and H5 (velocities VP5 and VS5).

\section{Quantifying uncertainties on the solution model: a linearized approach}

A classical approach to quantify uncertainties consists of the analysis of the Hessian matrix (or its inverse: the a posteriori covariance matrix) associated with the linearized problem (3) around the solution $m_{\infty}$. This approach is only valid in the vicinity of the solution model, the size of the vicinity depending on the nonlinearity of the forward map.

The bi-linear form associated with the Hessian matrix measures the influence of a model perturbation $\delta m$ on the quadratic cost function defined around the solution $m_{\infty}$ :

$$
\begin{aligned}
\tilde{C}(\delta m)-\tilde{C}(0)= & \frac{1}{2}\left(J_{\infty} \delta m\right)^{T} C_{D}^{-1}\left(J_{\infty} \delta m\right)+\frac{1}{2} \delta m^{T} C_{M}^{-1} \delta m \\
& -\delta m^{T}\left(J_{\infty}^{T} C_{D}^{-1} \delta T^{\mathrm{obs}}\left(m_{\infty}\right)-C_{M}^{-1} m_{\infty}\right) \\
= & \frac{1}{2} \delta m^{T}\left(J_{\infty}^{T} C_{D}^{-1} J_{\infty}+C_{M}^{-1}\right) \delta m \\
= & \frac{1}{2} \delta m^{T} H\left(m_{\infty}\right) \delta m .
\end{aligned}
$$

with

- $J_{\infty}=J\left(m_{\infty}\right)$

- $\tilde{C}(0)=C\left(m_{\infty}\right)$, and

- $J_{\infty}^{T} C_{D}^{-1} \delta T^{\mathrm{obs}}\left(m_{\infty}\right)-C_{M}^{-1} m_{\infty}=g\left(m_{\infty}\right)=0$, gradient of $C(m)$ that vanishes at the solution $m_{\infty}$.

The a posteriori covariance matrix is defined by the inverse of $H\left(m_{\infty}\right)$ (see [21])

$$
C_{M}^{\prime}=\left(J_{\infty}^{T} C_{D}^{-1} J_{\infty}+C_{M}^{-1}\right)^{-1}
$$

The space of admissible models can be characterized by the contour lines

$$
\left(m-m_{\infty}\right)^{T} C_{M}^{\prime-1}\left(m-m_{\infty}\right)=\text { constant }
$$

which are ellipsoids of centre $m_{\infty}$ and correspond also to contour lines of the a posteriori Gaussian probability density function

$$
\exp \left(-\frac{1}{2} \delta m^{T} C_{M}^{\prime-1} \delta m\right) \text {. }
$$


The diagonal terms of $C_{M}^{\prime}$ are the uncertainties on the parameters describing the model and the off-diagonal terms are the correlations between these uncertainties. For instance, the probability that the true model parameter $p_{i}$ verifies $-2\left(C_{M}^{\prime}\right)_{i, i} \leqslant p_{i}-p_{\infty} \leqslant 2\left(C_{M}^{\prime}\right)_{i, i}$, independently of the values of the other model parameters, is about $95 \%$. To take into account the correlations between the parameters, we should study the $95 \%$ confidence ellipsoid. The axes of the ellipsoids (6) are defined by the eigenvectors of $C_{M}^{\prime}$, the square root of the eigenvalues giving the uncertainties on the associated eigenvector.

Remark 1. In practice, the diagonal terms of $C_{M}^{\prime}$ provide the uncertainties on the B-spline parameters, unknowns of the discretized inverse problem: they are not physical quantities. We would rather compute uncertainties on physical quantities, for instance, the evaluation of the B-spline functions in the physical domain.

Remark 2. Following [8], the eigenvector decomposition of the a posteriori covariance matrix gives access to the worst/best determined model directions and the associated uncertainties. The best (respectively worst) determined model direction corresponds to the smallest (respectively highest) eigenvalue of the a posteriori covariance matrix, i.e. highest (respectively smallest) perturbation of the quadratic cost function. The uncertainties associated with these eigenvectors, namely the square root of the eigenvalues, are meaningless, the eigenvectors being composed of mixed velocity and interface parameters, combinations which are usually difficult to link to physical quantities.

From those remarks, we propose in the next sections two methods to quantify geological uncertainties on the solution model which avoid the expensive computation and the cumbersome analysis of the generally huge a posteriori covariance matrix $C_{M}^{\prime}$.

\subsection{Simulations of admissible models}

The first proposed method to quantify uncertainties is the simulation of admissible models from the a posteriori probability density function (pdf) (7). The simulations provide directly interpretable results, i.e. physical models. The method (see for instance [19]) consists in random simulations of model perturbations following the pdf (7).

First, we apply the variable transformation:

$$
\delta m^{\prime}=U \delta m,
$$

where $U$ is the lower triangular matrix obtained by a Cholesky decomposition of the inverse of the a posteriori covariance matrix $C_{M}^{-1}=H=U^{T} U$ (symmetric positive definite matrix).

The method consists then in simulations of the uncorrelated Gaussian pdf with unit variance

$$
\exp \left(-\frac{1}{2} \delta m^{\prime T} \delta m^{\prime}\right)
$$

Simulations $\delta m^{\prime}$ are transformed into correlated Gaussian simulations of vector $\delta m$ by the inverse transformation of (8).

Figures 3-7 show 100 simulated models obtained by this method from the solution model of figure 1: interfaces $H 4$ and $H 5$, variations of velocities VP5 and VS5 along $x$ and $z$ directions and histograms of the anisotropy parameters $\eta$ and $\delta$. From these simulations, we observe that the highest uncertainties on the lateral velocity variations are located at the boundaries of the model, areas that are not well illuminated by the rays. Indeed, for a slice at constant $z=2.7 \mathrm{~km}$, we observe uncertainties of $\approx 690 \mathrm{~m} \mathrm{~s}^{-1}$ for $V P 5$ and of $\approx 260 \mathrm{~m} \mathrm{~s}^{-1}$ for VS5 at the boundaries of the model and uncertainties of $\approx 450 \mathrm{~m} \mathrm{~s}^{-1}$ for VP5 and of $\approx 140 \mathrm{~m} \mathrm{~s}^{-1}$ for VS5 in the illuminated parts of the model. Concerning the interface depths, 


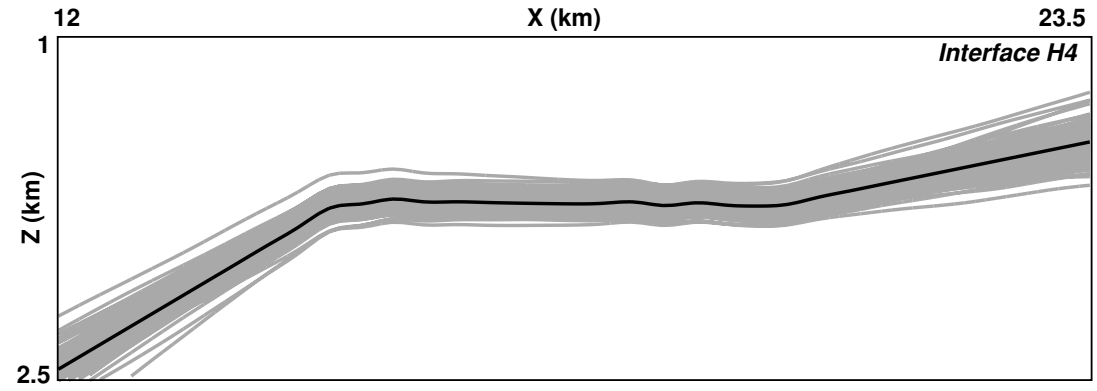

Figure 3. Reflector $H 4$ extracted from the 100 simulated velocity models. The bold line represents the solution interface.

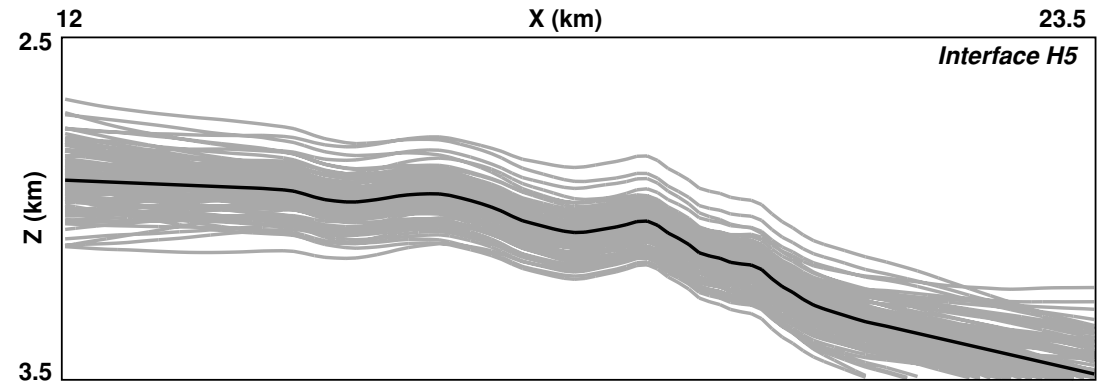

Figure 4. Reflector $H 5$ extracted from the 100 simulated velocity models. The bold line represents the solution interface.
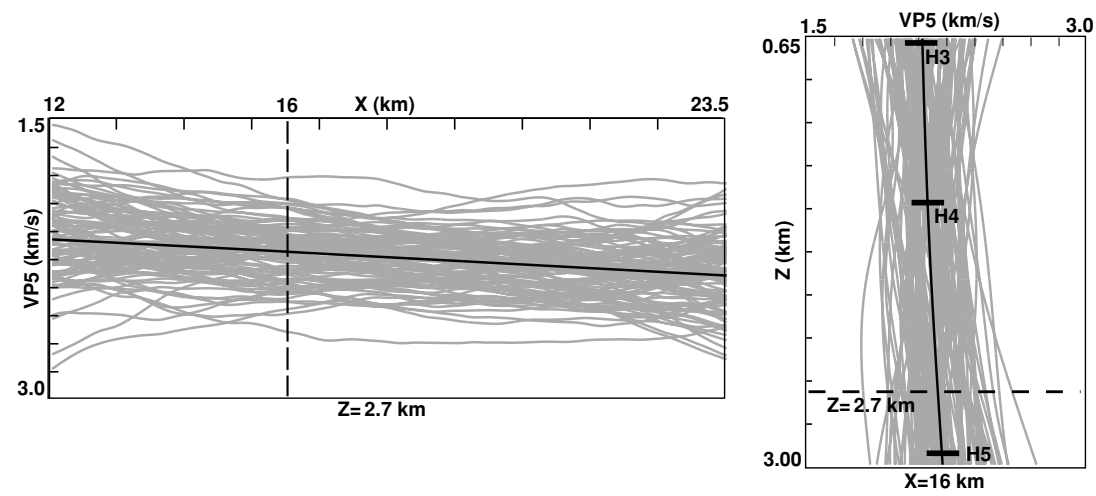

Figure 5. P-velocity variations along the $x$ axis at $z=2.7 \mathrm{~km}$ (left) and along the $z$ axis at $x=$ $16 \mathrm{~km}$ (right) extracted from the 100 simulated velocity models.

we observe uncertainties of $\approx 180 \mathrm{~m}$ for reflector $H 4$ and of $\approx 220 \mathrm{~m}$ for reflector $H 5$ at the boundaries and of $\approx 90 \mathrm{~m}$ for reflector $H 4$ and of $\approx 100 \mathrm{~m}$ for reflector $H 5$ elsewhere. For the anisotropy parameters $\eta$ and $\delta$, we notice uncertainties around $0.3 \%$ on $\eta$ and $2 \%$ on $\delta$.

This method is quite attractive for the straightforward interpretation of the results despite its cost (cost of the Cholesky decomposition): it provides physical models leading to small perturbations of the quadratic cost functions (3). 

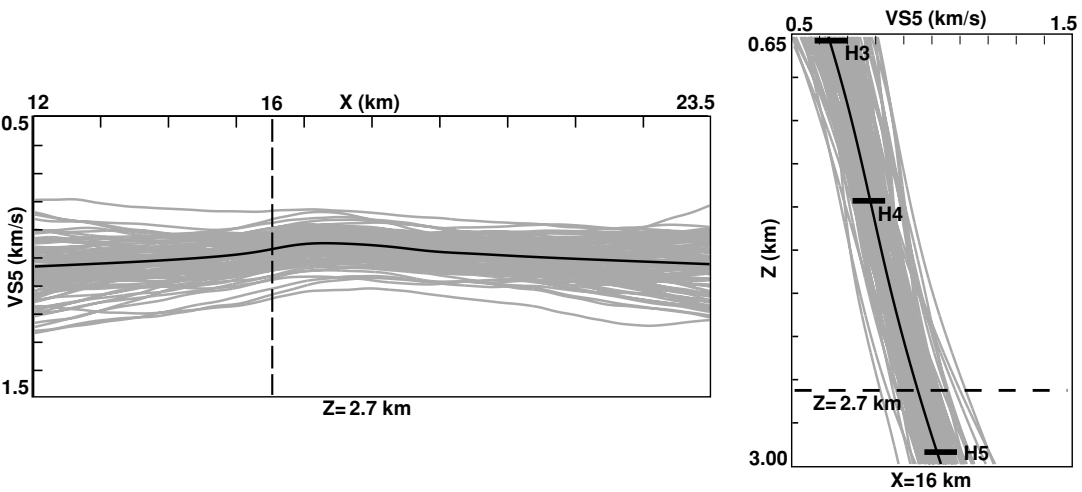

Figure 6. S-velocity variations along the $x$ axis at $z=2.7 \mathrm{~km}$ (left) and along the $z$ axis at $x=$ $16 \mathrm{~km}$ (right) extracted from the 100 simulated velocity models.
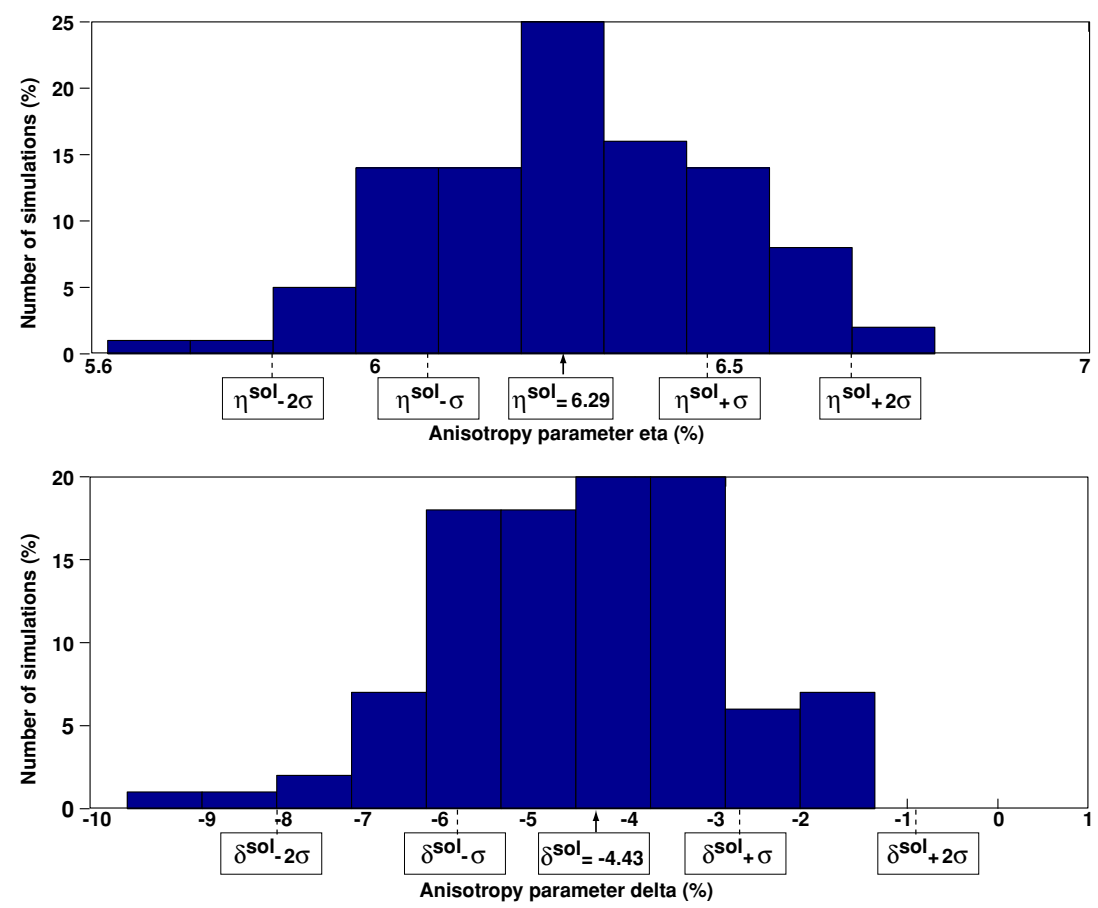

Figure 7. Histograms of the 100 simulated velocity models. Top: anisotropy parameter $\eta$. Bottom: anisotropy parameter $\delta . \sigma$ corresponds to the standard deviation on the anisotropy parameter $\eta$ (respectively $\delta$ ) given by the macro-parameter method (see section 1 ).

\subsection{Uncertainties on geological macro-parameters}

We propose here a method that allows us to deal with large size models at a reasonable cost and provides uncertainties on chosen physical quantities. The proposed approach consists in building macro-parameters (MP) with a geophysical interest. These macro-parameters are linear combinations of the inverted parameters such as the mean of the velocity variations in a zone, the slope of an interface, the average thickness of a layer, etc. [12] has introduced the notion of macro-parameter (his main motivation being to avoid numerical problems in the 
Table 1. Standard deviations in bold (square roots of the diagonal terms of the a posteriori covariance matrix) associated with the MP 'mean in the illuminated part of the model' and the normalized correlations between the uncertainties on the different defined MP. This uncertainty analysis is performed for the solution model of figure 1 . The normalized values $\frac{\sigma}{\mid \text { value }^{\mathrm{sol} \mid}} \times 100$ of the standard deviations $(\sigma)$ are also indicated in italic.

\begin{tabular}{lllllll}
\hline & $V P 5$ & $V S 5$ & $\eta$ & $\delta$ & $H 4$ & $H 5$ \\
\hline$V P 5$ & $\mathbf{4 7 5 . 1} \mathbf{~ m ~ s}^{-1}(22 \%)$ & 0.002 & -0.03 & -0.02 & 0.005 & 0.01 \\
$V S 5$ & 0.002 & $\mathbf{1 6 8 . 9} \mathbf{~ m ~ s}^{-1}(22 \%)$ & -0.04 & -0.03 & 0.005 & 0.01 \\
$\eta$ & -0.03 & -0.04 & $\mathbf{0 . 2 2} \%(4 \%)$ & $\underline{0.93}$ & -0.16 & $\underline{-0.33}$ \\
$\delta$ & -0.02 & -0.03 & $\underline{0.93}$ & $\mathbf{1 . 6} \%(37 \%)$ & -0.17 & $\underline{-0.36}$ \\
$H 4$ & 0.005 & 0.005 & -0.16 & -0.17 & $\mathbf{7 7 . 1} \mathbf{~ m}(5 \%)$ & 0.06 \\
$H 5$ & 0.01 & 0.01 & $\underline{-0.33}$ & $\underline{-0.36}$ & 0.06 & $\mathbf{8 0 . 3} \mathbf{~ m ( 3 \% )}$ \\
\hline
\end{tabular}

inversion of the complete Hessian). We propose here a generalization of his work (general definition of macro-parameters) which allows the computation of uncertainties for huge 3D problems.

We define a macro-parameter as

$$
P=B p,
$$

where $p$ is the $n_{p}$ model parameters vector (B-spline parameters in our case), $P$ is the $n_{\mathrm{MP}}$ macro-parameters vector and $B$ is the condensation matrix. We compute the a posteriori covariance matrix $\tilde{C}_{M}^{\prime}$ in the macro-parameter space:

$$
\tilde{C}_{M}^{\prime}=B C_{M}^{\prime} B^{T} \text {. }
$$

Note that $\tilde{C}_{M}^{\prime}$ (an $n_{\mathrm{MP}} \times n_{\mathrm{MP}}$ matrix) is small compared to $C_{M}^{\prime}$ (an $n_{p} \times n_{p}$ matrix) since $n_{\mathrm{MP}} \ll n_{p}$. To obtain this matrix, we do not need to compute the whole inverse of the Hessian, $C_{M}^{\prime}=H^{-1}$, in the parameter space.

Indeed, to obtain $\tilde{C}_{M}^{\prime}=\tilde{H}^{-1}=B H^{-1} B^{T}$ in the MP space, we just need $H^{-1} B_{j}^{T}$, where $B_{j}^{T}$ are the different columns of $B^{T}$. We thus solve $n_{\mathrm{MP}}$ linear systems, $\tilde{H}_{j}^{-1}=B H^{-1} B_{j}^{T}$, that are similar to the linearized problem we solve at each Gauss-Newton iteration. Thus, the computational cost for one MP is comparable to one iteration of the inversion process.

We applied this method on the model of figure 1. We choose simple MP: the mean of the velocity variations and the mean of the interface depth in the illuminated part of the layer (region covered by rays) ${ }^{7}$. The results are listed in table 1 . The uncertainty on anisotropy parameter $\delta$ is high: $\pm 74 \%$ (if we consider twice the relative standard deviation) and we observe high correlations between $\eta$ and $\delta(0.93)$ and also correlations between anisotropy parameters and $H 5$ depth $(-0.33$ and -0.36$)$. We notice also the bad determination of the velocities: $\pm 22 \%$ (relative value). All these results are consistent with the simulation results (section 4.1).

This method with its general formalism allows us to compute uncertainties on relevant geological quantities with a reasonable computation cost and to highlight easily strong coupling between the chosen MP.

\subsection{Discussions on the two methods}

As already mentioned, the two methods described in sections 4.1 and 4.2 rely on the quadratic approximation (3) of the nonlinear cost function. In table 2 we have listed the RMS of traveltime misfits for 20 simulated models (computed by the ray tracing forward operator).

\footnotetext{
7 From figure 2, we define the illuminated region by $x \in[14,20.5 \mathrm{~km}]$.
} 

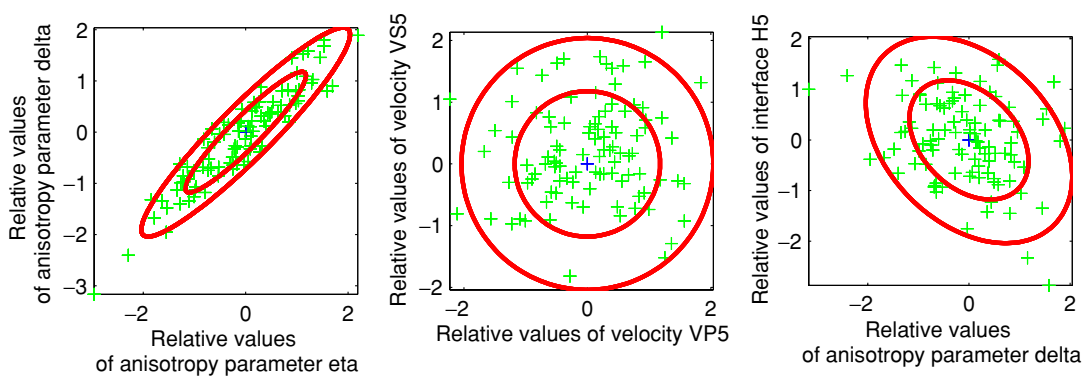

Figure 8. $68 \%$ and $95 \%$ isovalues of the marginal probability density function for the anisotropy parameters $\eta$ and $\delta$ (left), P-velocity and S-velocity (middle), $\delta$ and the interface depth of reflector h5 (right). The represented values are the relative ones (for instance, $\frac{\eta-\eta^{\text {sol }}}{\left|\sigma_{\eta}\right|}$, where $\sigma_{\eta}$ is the standard deviation for the anisotropy parameter $\eta$ ). The crosses represent the MP values of the 100 simulated models.

Table 2. RMS values of the traveltime misfits, mean value of the velocities VP5 and VS5, of the interfaces $H 4$ and $H 5$ in the illuminated region (MP defined in section 4.2 and anisotropy parameters $\eta$ and $\delta$ for 20 simulated models (chosen among the 100 simulations presented in section 4.1).

\begin{tabular}{llllllll}
\hline Models & RMS $(\mathrm{ms})$ & $V P 5\left(\mathrm{~km} \mathrm{~s}^{-1}\right)$ & $V S 5\left(\mathrm{~km} \mathrm{~s}^{-1}\right)$ & $H 4(\mathrm{~km})$ & $H 5(\mathrm{~km})$ & $\eta(\%)$ & $\delta(\%)$ \\
\hline 1 & 6.7 & 3.736 & 1.154 & 1.783 & 3.131 & 6.5 & -2.6 \\
2 & 6.6 & 3.121 & 1.222 & 1.925 & 3.222 & 6.2 & -4.9 \\
3 & 6.4 & 2.746 & 1.102 & 1.790 & 3.060 & 6.0 & -5.9 \\
4 & 6.9 & 2.701 & 1.315 & 1.857 & 3.259 & 6.2 & -3.7 \\
5 & 6.2 & 3.300 & 1.416 & 1.817 & 3.142 & 5.7 & -8.3 \\
6 & 6.5 & 2.542 & 1.245 & 1.926 & 3.181 & 6.1 & -4.6 \\
7 & 6.6 & 2.536 & 1.137 & 1.925 & 3.143 & 6.1 & -5.5 \\
8 & 6.2 & 1.919 & 1.087 & 1.844 & 3.102 & 6.3 & -4.8 \\
9 & 6.2 & 3.182 & 1.078 & 1.875 & 3.267 & 6.5 & -3.5 \\
10 & 6.4 & 2.960 & 1.388 & 1.736 & 3.198 & 6.0 & -6.2 \\
11 & 6.2 & 3.247 & 1.452 & 1.815 & 3.173 & 6.0 & -6.4 \\
12 & 6.2 & 2.592 & 1.073 & 1.664 & 3.147 & 6.6 & -1.4 \\
13 & 6.5 & 2.510 & 1.239 & 1.817 & 3.239 & 6.2 & -4.9 \\
14 & 6.6 & 3.595 & 1.130 & 1.717 & 3.208 & 6.5 & -1.9 \\
15 & 11.6 & 2.344 & 1.172 & 1.770 & 3.144 & 6.0 & -6.1 \\
16 & 6.5 & 2.154 & 1.323 & 1.853 & 3.2322 & 6.3 & -4.5 \\
17 & 6.3 & 2.855 & 1.307 & 1.797 & 3.296 & 6.3 & -3.4 \\
18 & 6.2 & 2.125 & 1.049 & 1.921 & 3.118 & 5.9 & -6.8 \\
19 & 6.6 & 1.431 & 1.048 & 1.772 & 3.144 & 6.4 & -3.7 \\
20 & 6.3 & 2.371 & 1.225 & 1.864 & 3.166 & 6.0 & -6.6 \\
\hline
\end{tabular}

Note that the RMS traveltime misfit for the solution model is $6.2 \mathrm{~ms}$. Thus, there is only one model which is not acceptable (11.6 ms for the model 15). The others are admissible models with a RMS traveltime misfit bounded by $7 \mathrm{~ms}$. The simulation method has then provided model perturbations that correspond to small perturbations of the quadratic cost function but also to small perturbations of the nonlinear cost function (RMS of traveltime misfits remains small). It shows for this example, the good agreement between the quadratic cost function and the nonlinear one around the solution model.

Figure 8 illustrates isovalues of the probability density function (7) in the MP space, i.e. the ellipsoids defined by $\delta m_{\mathrm{MP}}^{T} \tilde{C}_{M}^{\prime-1} \delta m_{\mathrm{MP}}$. We visualize the $2 \mathrm{D}$-marginal probability 

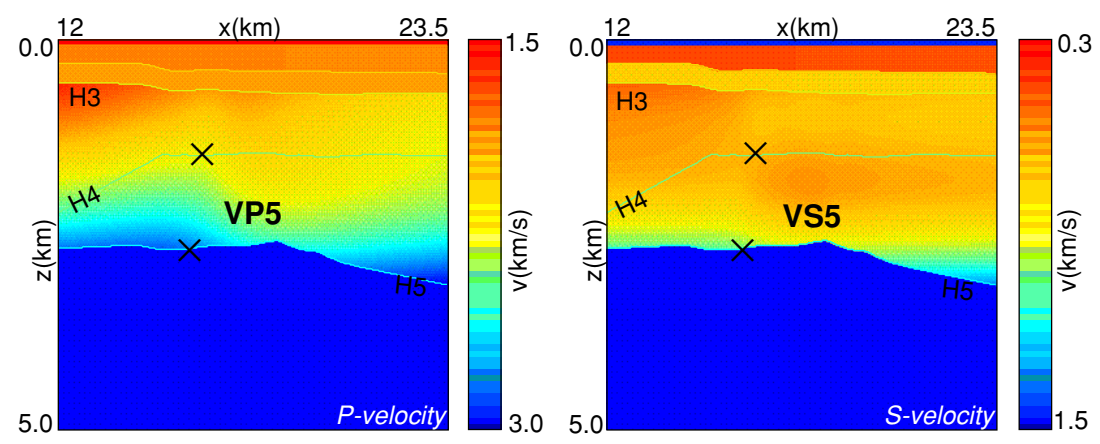

Figure 9. Solution model 2 obtained by tomography with additional constraints on the interface depth at well locations. The RMS value of the traveltime misfits is $6.0 \mathrm{~ms}$. The anisotropy parameters values are $\eta=8.27 \%$ and $\delta=15.94 \%$. The crosses represent the wells.

density functions for several relevant MP couples, the $68 \%$ confidence ellipsoid and the $95 \%$ confidence ellipsoid for $\eta$ and $\delta, V P 5$ and VS5, $\delta$ and $H 5$. This representation allows us to underline the correlations between the anisotropy parameters $\eta$ and $\delta$ which are quite strong in this example. The $68 \%$ and the $95 \%$ confidence ellipsoids are elongated (the left part of figure 8). On the opposite, the $\mathrm{P}$ and $\mathrm{S}$ velocities are uncorrelated: we obtain almost a circle (the middle part of figure 8 ). We can also observe correlations between the anisotropy parameter $\delta$ and the mean of the last reflector depth $H 5$ (the right part of figure 8). In this figure, we have also superimposed the anisotropy parameters $\eta$ and $\delta$ and the MP VP5 and $V S 5, \delta$ and MP $H 5$ built from the 100 simulated models. This figure allows us to point out the equivalence of the simulation method and the macro-parameter approach.

Remark that the macro-parameter approach provides interesting information on the correlations of macro-parameter uncertainties and has a lower computation cost than simulations of the model parameters using the complete a posteriori covariance matrix. We could also perform Gaussian simulations of the macro-parameters using the reduced $a$ posteriori covariance matrix.

\section{Nonlinear a posteriori analysis}

We have shown in the previous sections that a linearized uncertainty analysis allows us to delimit the range of possible solution models that fit, with the expected accuracy, the data and the a priori information. Nevertheless, we should keep in mind that this approach is only valid in the vicinity of the solution model (linearized framework) and complex cases may require a nonlinear approach.

Model 15 of table 2 indicates that some of the simulated models may produce unacceptable traveltimes misfits $(\mathrm{RMS} \approx 11 \mathrm{~ms}$ and $\mathrm{MAX}=25 \mathrm{~ms}$ for PP data and $=41 \mathrm{~ms}$ for PS data). It shows the limitation of the quadratic approximation of the nonlinear cost function and thus the limitation of the linearized methods of uncertainty quantification. Moreover, [7] has shown that a tomographic inversion with constraints on the location of the interfaces at well locations provided a very different model (model 2 shown in figure 9) from the solution model of figure 1 . The two models verify the traveltime data with the expected accuracy. This second model does not belong to the range of possible models detected by the two methods we proposed: indeed, $\delta$ is equal to $15.94 \%$ whereas the expected range of values was between $-8 \%$ and $-1 \%$ (see for instance figure 7 ). It shows again the limitations of the linearized approach. 

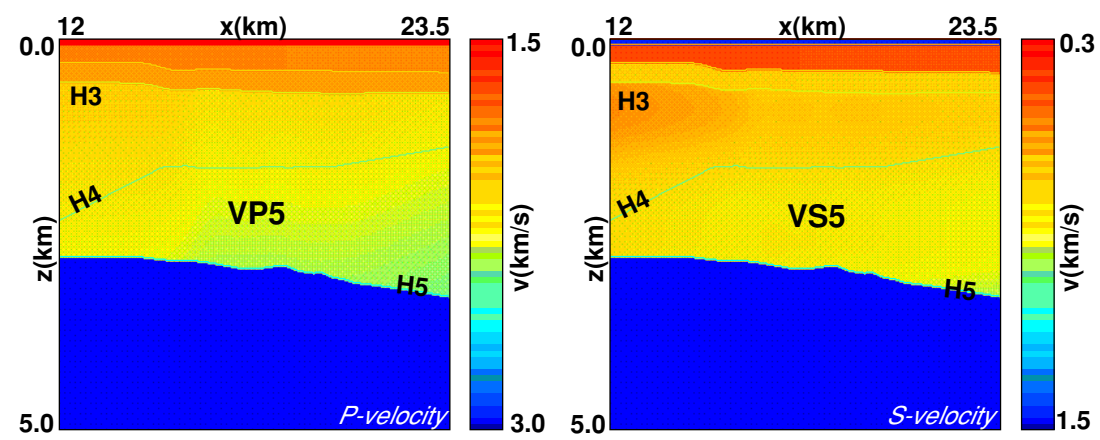

Figure 10. Solution model 3 obtained by tomography with a constrain on $\delta$ parameter to remain positive. The RMS value of the traveltime misfits is $6.371 \mathrm{~ms}$. The anisotropy parameters values are $\eta=6.2 \%$ and $\delta=2 \%$.

Table 3. Computational times (platform: Silicon Graphics Fuel V10 Graphics $500 \mathrm{MHz}$ $\mathrm{R} 14000 \mathrm{~A}$ ) for unconstrained inversion, constrained inversion, Cholesky factorization and one macro-parameter (MP).

\begin{tabular}{|c|c|c|c|c|}
\hline & $\begin{array}{l}\text { Unconstrained } \\
\text { inversion }\end{array}$ & $\begin{array}{l}\text { Constrained } \\
\text { inversion }\end{array}$ & $\begin{array}{l}\text { Cholesky } \\
\text { factorization }\end{array}$ & $\begin{array}{l}\text { Macro-parameter } \\
\text { (MP) }\end{array}$ \\
\hline $\begin{array}{l}\mathrm{CPU} \\
\text { time }\end{array}$ & $\begin{array}{l}1797 \mathrm{~s} / \text { iteration } \\
\text { with } 1779 \mathrm{~s} / \text { iteration } \\
\text { for ray tracing }\end{array}$ & $\begin{array}{l}1930 \mathrm{~s} / \text { iteration } \\
\text { with } 1779 \mathrm{~s} / \text { iteration } \\
\text { for ray tracing }\end{array}$ & $\begin{array}{l}9251 \mathrm{~s} \\
\text { for } 100 \text { simulated } \\
\text { models without } \\
\text { raytracing }\end{array}$ & $\begin{array}{l}1684 \mathrm{~s} / \mathrm{MP} \\
\text { without } \\
\text { ray tracing }\end{array}$ \\
\hline
\end{tabular}

Table 4. Three different solution models. Model 1, model 2 and model 3 are, respectively, the models of figures 1,9 and 10 .

\begin{tabular}{lcll}
\hline & Model 1 & Model 2 & Model 3 \\
\hline Mean of the P-velocity & $2.849 \mathrm{~km} \mathrm{~s}^{-1}$ & $3.623 \mathrm{~km} \mathrm{~s}^{-1}$ & $2.895 \mathrm{~km} \mathrm{~s}^{-1}$ \\
Mean of the S-velocity & $1.229 \mathrm{~km} \mathrm{~s}^{-1}$ & $1.621 \mathrm{~km} \mathrm{~s}^{-1}$ & $1.213 \mathrm{~km} \mathrm{~s}^{-1}$ \\
$\eta$ & $6.29 \%$ & $8.27 \%$ & $6.2 \%$ \\
$\delta$ & $-4.4 \%$ & $15.94 \%$ & $2 \%$ \\
Mean of the depth of the reflector $H 4$ & $1.838 \mathrm{~km}$ & $1.755 \mathrm{~km}$ & $1.659 \mathrm{~km}$ \\
Mean of the depth of the reflector $H 5$ & $3.167 \mathrm{~km}$ & $3.021 \mathrm{~km}$ & $2.882 \mathrm{~km}$ \\
\hline
\end{tabular}

To perform a nonlinear analysis we have chosen an experimental approach which consists of performing several constrained inversions allowing us to test different geological scenarii to try to delimit the space of admissible solutions. A sequential quadratic programming (SQP) approach based on an augmented Lagrangian method (for details see [7]) is used to solve this nonlinear constrained optimization problem (computational times listed in table 3 ).

For instance, we could test different hypotheses on the values of the anisotropy parameters for which a strong uncertainty has been detected by the linearized approach. If we introduce a constraint on the anisotropy parameter $\delta$, such as $\delta>0$, we find model 3 displayed in figure 10: this result expands the range of admissible models (variability of $\delta$ detected by the MP approach was $-4.43 \pm 3.2 \%$ ). 
In the same way, we can test the variability of the anisotropy parameter $\eta$. We note (see table 4 ) that the value of the anisotropy parameter $\eta$ of model 2 (figure 9) is $8.27 \%$, but all the simulated values of this parameter are around $6 \%$ and the variability of $\eta$ provided by the MP is $\pm 0.44 \%$. Nevertheless, tomographic inversion under the constraint $\eta \leqslant 4 \%$ or $\eta \geqslant 8.5 \%$ does not provide a model that satisfies both these constraints and the data with the expected accuracy.

We have shown that, by constrained tomography, we can test different geological hypotheses and delimit the space of admissible models and go further than the linearized approach which explores only the vicinity of the solution model which may be one local minimum around others.

\section{Conclusions-discussions}

Reflection tomography provides the velocity model that best fits the traveltime data. However, this solution is only one among many admissible models. An a posteriori uncertainty analysis is crucial to delimit the range of possible solution models that would fit the data and the a priori information with the expected accuracy. In this paper, we describe two methods, the simulations and the macro-parameter approach, to perform a linearized a posteriori analysis, approach valid only in the vicinity of the solution model. The simulation method based on the analysis of the a posteriori covariance matrix is quite attractive for the straightforward interpretation of the results despite its cost. We propose a general formalism to reduce the a posteriori analysis to geological quantities of the model and thus reduce the cost of the uncertainty estimation. These methods give interesting results on a $2 \mathrm{D}$ real data set. These methods being valid only in the vicinity of the solution we propose to complete the linearized study by a nonlinear approach based on constrained optimization.

\section{Acknowledgments}

The authors would like to thank BP for providing the data set, Karine Broto, Frédéric Delbos, Andréas Ehinger, Anne Jardin, Patrick Lailly and Timothée Perdrizet for many fruitful discussions.

\section{References}

[1] Berryman J G 2000 Analysis of approximate inverses in tomography: II. Iteratives inverses Optim. Eng. $1437-73$

[2] Bishop T N, Bube K P, Cutler R T, Langan R T, Love P L, Resnick J R, Shuey R T, Spindler D A and Wyld H W 1985 Tomographic determination of velocity and depth in laterally varying media Geophysics 50 903-23

[3] Broto K, Ehinger A and Kommedal J 2003 Anisotropic traveltime tomography for depth consistent imaging of PP and PS data The Leading Edge 22 114-9

[4] Bube K P and Langan R T 1994 A continuation approach to regularization for traveltime tomography Expanded Abstracts 64th Annual International Meeting Society Exploration Geophysicists pp 980-3

[5] Červený V 1987 Raytracing algorithms in three-dimensional laterally varying layered structures Seismic Tomography ed G Nolet (Dordrecht: Reidel) pp 99-133

[6] de Boor C 1978 A Practical Guide to Splines (Berlin: Springer)

[7] Delbos F, Gilbert J Ch, Glowinski R and Sinoquet D 2005 Constrained optimization in seismic reflection tomography: a Gauss-Newton augmented Lagrangian approach Geophys. J. Int. 164 670-84

[8] Delprat-Jannaud F and Lailly P 1992 What information on the earth model do reflection travel times hold? $J$. Geophys. Res. 97 19827-44

[9] Delprat-Jannaud F and Lailly P 1993 Ill-posed and well-posed formulations of the reflection traveltime tomography problem J. Geophys. Res. 98 6589-605 
[10] Duffet C and Sinoquet D 2002 Quantifying geological uncertainties in velocity model building Expanded Abstracts, 72nd Annual International Meeting Society Exploration Geophysicists pp 926-9

[11] Gouveia W P and Scales J A 1997 Resolution of seismic waveform inversion: Bayes versus Occam Inverse Problems 13 323-49

[12] Grenié D 2001 Tomographie de temps de trajet adaptée au problème de l'imagerie 3D en exploration pétrolière: théorie, mise en oeuvre logicielle et applications PhD Thesis Université Paris VII

[13] Inoue H 1986 A least-squares smooth fitting for irregularly spaced data: finite element approach using the cubic B-splines basis Geophysics $\mathbf{5 1} 2051-66$

[14] Jurado F, Sinoquet D and Ehinger A 1996 3D reflection tomography designed for complex structures Expanded Abstracts, 66th Annual International Meeting Society Exploration Geophysicists pp 711-4

[15] Jurado F, Lailly P and Ehinger A 1998 Fast 3D two-point ray tracing for traveltime tomography Proc. SPIE $345370-81$

[16] http://consortium.ifp.fr/KIM/ 2001 Web pages of former KIM consortium

[17] Malinverno A and Leaney S 2000 A Monte Carlo method to quantify uncertainty in the inversion of zero-offset VSP data 70th Annual Meeting Society Exploration Geophysicists

[18] Mosegaard K and Tarantola A 1995 Monte Carlo sampling of solutions to inverse problems J. Geophys. Res. $100431-47$

[19] Parker R 1994 Geophysical Inverse Theory (Berlin: Springer)

[20] Stopin A 2001 Reflection tomography of compressional and shear modes for determination of anisotropic velocity models $P h D$ Thesis Université Louis Pasteur Strasbourg I

[21] Tarantola A 2004 Inverse Problem Theory (Philadelphia, PA: SIAM) p 342

[22] Thomsen L 1986 Weak elastic anisotropy Geophysics 51 1954-66 